\title{
Analisis Pesta para la consolidación de la cadena productiva forestal en el Departamento de Arauca
}

\author{
Pesta analysis for consolidation \\ of the chain of forestry at Arauca Department
}

Recibido: 9 de febrero de 2015

Evaluado: 4 de abril de 2015

Aceptado: 2 de julio de 2015

\author{
Andrea Constanza Padilla Puerta (Colombia) \\ Maestría en Administración. Universidad Cooperativa de Colombia \\ acpadillap@unal.edu.co
}

\section{Luz Alexandra Montoya Restrepo (Colombia)}

Doctorado en Ciencias Económicas. Universidad Nacional de Colombia

lamontoyar@unal.edu.co

\section{Ivan Alonso Montoya Restrepo (Colombia)}

Doctorado en Ciencias Económicas. Universidad Nacional de Colombia

iamontoyar@unal.edu.co

\section{Resumen:}

La consolidación de cadenas productivas contribuyen al desarrollo de capacidades nacionales y locales para la producción, transformación y comercialización de bienes y servicios, enmarcado en la búsqueda de la productividad y la competitividad. El objetivo principal de esta investigación fue realizar un análisis del sector forestal del departamento de Arauca, usando información primaria, generada por los actores y expertos de la cadena, e información secundaria, generada por las entidades territoriales con el fin de identificar los factores clave que inciden directamente en el desarrollo de la cadena productiva forestal en el Departamento, así como sus actores. Para ello, se desarrolló un ejercicio prospectivo a fin de estructurar una propuesta para la consolidación de la cadena forestal del departamento de Arauca, acorde con las potencialidades y particularidades locales.

Palabras Clave: Análisis Pesta, cadena productiva, eslabones, Plantaciones forestales comerciales, sostenibilidad ambiental.

\section{Summary:}

The consolidation of productive chains contribute to the development of national and local capacities for production, processing and marketing of goods and services, whose action is framed in the pursuit of productivity and competitiveness. The main objective of this research was to analyze the the forestry in the department of Arauca, used primary data generated by the actors and experts in the chain, and secondary data generated by local institutions. For identify the key factors that directly affect the development of the forest production chain in the Department, were consulted with the stakeholders and represents of institutions, with whom a prospective exercise was conducted, the results present a proposal consistent with potentials and local characteristics, and aims to provide elements for the consolidation of the chain in the department of Arauca.

Keywords: commercial forest plantations, environmental sustainability, links of the chain production, Pesta analysis, productive chain. 


\section{Introducción}

Los bosques, siendo uno de los ecosistemas que generan una gran cantidad de bienes y servicios, han empezado a ser percibidos como una fuente para la solución de las problemáticas actuales, donde el concepto de desarrollo sostenible cobra más fuerza, convirtiéndose éstos en un componente importante en la toma de decisiones y en la política económica de los Estados; sin embargo, es claro que "las actividades de desarrollo solo serán sostenibles si consiguen equilibrar los factores económicos, sociales y ecológicos" y, para ello, "es necesario promover la armonía con la naturaleza”, estableciendo sistemas económicos de producción sostenible, al mismo tiempo que se da prioridad a la conservación ambiental y la gestión social en el territorio de influencia de los proyectos forestales. Debido al auge medio ambiental en el mundo, basado en estos recientes conceptos, se ha venido realizando la reorientación de los procesos económicos, enfocados en el aprovechamiento organizado de los recursos forestales de las regiones, más aún cuando son claros los bienes y servicios, tangibles e intangibles, obtenidos de los esquemas boscosos. Son reconocidos los aportes que estos ecosistemas realizan debido a que se constituyen en refugio y facilitan la conservación y recuperación de biodiversidad, contribuyen con la protección de cuencas hidrográficas, mejoran las condiciones de los suelos, facilitan la fijación de carbono y la producción de oxígeno, generan empleo y, en consecuencia, son aprovecha- dos por millones de personas que encuentran en ellos una forma de suplir sus necesidades de sustento, entre las innumerables externalidades positivas que se pueden estimar y, además, generan rendimientos económicos. Con este entorno el presente documento tiene como pregunta de investigación reconocer cuáles son los elementos de competitividad fundamentales a partir de un análisis PESTA (derivado del concepto PEST incluye la evaluación de los factores Políticos, económicos, sociales, tecnológicos y ambientales, (Aithal et al (2015), Shabanova et al (2015) y Ortiz et al (2015)) que le permitan a la cadena productiva fortalecerse para así competir en igual de condiciones en un mercado que cada vez es más exigente.

\section{Marco Conceptual}

Según la FAO, "América Latina y el Caribe representan alrededor del $7 \%$ del valor del sector forestal mundial y el $18 \%$ del valor ańadido del sector de los bosques primarios y el $6 \%$ de la industria de pasta y papel"; (FAO, 2007, P.42) motivo por el cual es considerada como una importante fuente de materias primas para el desarrollo de procesos de transformación en productos terminados, los cuales se realizan en otras regiones del mundo. Dado el creciente interés por el sector forestal, la FAO identificó una serie de elementos que pueden incidir en el desarrollo de la industria forestal, entre los que se destacan factores internos y externos presentados en la (Tabla $1)$.

Tabla 1: Factores que inciden en el desarrollo de una industria forestal

\begin{tabular}{|c|c|}
\hline FACTORES EXTERNOS & INCIDENCIA \\
\hline $\begin{array}{l}\text { Evolución demográfica y el crecimiento } \\
\text { económico }\end{array}$ & $\begin{array}{l}\text { Pueden incidir en la demanda de productos forestales y en la oferta de nuevos productos } \\
\text { y/o servicios a nuevos compradores, así como en el crecimiento de mercados internos y el } \\
\text { aumento de la producción para exportación. }\end{array}$ \\
\hline Cambios en los sectores competidores & $\begin{array}{l}\text { El establecimiento de relaciones sinérgicas de trabajo favorecerían la industria y sus } \\
\text { asociadas; sin embargo, es importante tener en cuenta no solo a los competidores actuales; } \\
\text { sino también a los potenciales, dado que en algún momento puede cambiar el mercado y } \\
\text { favorecer a otras industrias. }\end{array}$ \\
\hline Tendencias sociales & $\begin{array}{l}\text { Las percepciones de los consumidores sobre productos van más allá de su costo y } \\
\text { características funcionales, también incluyen factores intangibles que satisfacen otras } \\
\text { necesidades. De la misma manera, el nivel de educación de los consumidores, así como } \\
\text { el acceso a medios globales de comunicación influyen. }\end{array}$ \\
\hline $\begin{array}{l}\text { Competencia por los recursos (tierras, } \\
\text { mano de obra y capital) }\end{array}$ & Influye en la oferta en la medida en que se consigan o no. \\
\hline $\begin{array}{l}\text { Cambios en la propiedad, el control y la } \\
\text { ordenación de los bosques }\end{array}$ & $\begin{array}{l}\text { La disponibilidad de tierra para el desarrollo de proyectos forestales afecta de manera directa } \\
\text { la oferta de bienes y servicios. }\end{array}$ \\
\hline $\begin{array}{l}\text { Madurez de los actuales mercados de } \\
\text { productos }\end{array}$ & $\begin{array}{l}\text { Generada por la concentración de la demanda en unos pocos usos finales, considerándose } \\
\text { algunos como maduros, resultando difícil desarrollar procesos de innovación en este tipo de } \\
\text { mercados. }\end{array}$ \\
\hline $\begin{array}{l}\text { Propiedades ambientales de los produc- } \\
\text { tos forestales }\end{array}$ & $\begin{array}{l}\text { Los productos forestales, por ser de origen natural y con huella de carbono baja, pueden } \\
\text { preferirse frente a otros existentes en el mercado, considerados como sustitutos. }\end{array}$ \\
\hline Estructura e inversiones de la industria & $\begin{array}{l}\text { La industria forestal suele estar fragmentada y repartida en un país, sin que existan } \\
\text { cohesiones, ni trabajo conjunto, ni capacitación e inversión en tecnología y, todo esto, bajo } \\
\text { un manto constante de informalidad. }\end{array}$ \\
\hline
\end{tabular}




\begin{tabular}{|l|l|}
\hline FACTORES EXTERNOS & INCIDENCIA \\
\hline $\begin{array}{l}\text { Costo de mano de obra y condiciones } \\
\text { laborales }\end{array}$ & $\begin{array}{l}\text { A pesar de la tendencia hacia la mecanización, aún se sigue requiriendo mano de obra sobre } \\
\text { todo en los procesos de producción y transformación a pequeña escala. Aunque existen } \\
\text { trabajos repetitivos y sin exigencia académica, también existen oportunidades de generación } \\
\text { de procesos y productos creativos e innovadores en etapas de diseño y comercialización. }\end{array}$ \\
\hline & $\begin{array}{l}\text { El aprovechamiento forestal, especialmente de zonas de bosque natural, tiene implicaciones } \\
\text { negativas para un gran número de personas, generando no solo problemas ambientales sino } \\
\text { también problemas sociales difíciles de resolver, lo que hace que en ocasiones se incrementen } \\
\text { los costos de inversión, para resolver los conflictos ambientales y mediar en los sociales, por } \\
\text { ejemplo, mediante la reserva de zonas forestales para conservación y restauración, aunque } \\
\text { también se pueden obtener ventajas con este tipo de soluciones, mediante la generación de } \\
\text { otros beneficios de los bosques. En otros casos, algunos sectores en la industria forestal } \\
\text { alcanzan altos niveles de desempeño ambiental y social, gracias a las exigencias de los } \\
\text { consumidores de productos. }\end{array}$ \\
\hline \multicolumn{2}{|c|}{ Fuente: Elaboración propia con información de la FAO (2011) }
\end{tabular}

Desde la entrada en vigor de la Ley 811 de 2003, se establece el marco para que en Colombia se avance en la organización de la cadena productiva del sector forestal (Tableros, aglomerados y contrachapados - Muebles y productos de madera).

Es así como "El Plan Nacional de Desarrollo Forestal (PNDF) propone como meta el establecimiento de 1'500.000 hectáreas en plantaciones productoras para el año 2025" (SIAC, 2015, p.1), ya que es necesario fortalecer este sector en el país, "según información reportada por la FAO, Colombia ocupa el puesto 41 como productor de madera en rollo industrial en el mundo y participa con apenas el $0,2 \%$ del total de la producción mundial" (Productos de Colombia, 2015).

Es así como, "de las 114 millones de hectáreas de extensión del país, existe un potencial para el desarrollo de proyectos forestales de 17 millones de hectáreas, de las cuales sólo están siendo utilizadas en plantaciones forestales comerciales 350.000 has" (Proexport, 2012, p. 4).

El impacto del sector en Colombia es aún inicipiente, el cual representa:

"únicamente entre el $0.4 \%$ y el $0.5 \%$ del PIB, a través de la contribución de dos sectores, el silvícola y el manufacturero. El primero incluye la producción obtenida del aprovechamiento de bosques (naturales y plantados, la reforestación y los productos como látex, gomas, resinas, cortezas, madera en bruto y leña. El segundo incluye el sector de muebles y madera y la industria de pulpa el papel y el cartón" (Olarte, SF. P. 3)
Sin embargo, se estima que el país tiene 24'556.854 hectáreas aptas el establecimiento de plantaciones forestales comerciales:

\begin{abstract}
"constituyéndose en uno de los sectores con mayores posibilidades de crecimiento económico y social, además dadas las ventajas en oferta natural de bosques que tiene el país y los altos rendimientos que presentan algunas especies forestales en cultivos de tipo comercial".
\end{abstract}

A nivel de mercados, los productos de la cadena forestal en Colombia, tienen como destino principal el mercado doméstico y están representados en:
"madera en rollo, pulpa de madera, chapas y contrachapado, tableros aglomerados, además de muebles, es- tos últimos son producidos a nivel in- dustrial por tan solo siete (7) empre- sas con entre 350 y 500 empleados $\mathrm{y}$, a nivel sub-industrial y artesanal, por talleres de menos de cinco (5) empleados; p. 22"

En los últimos años, en la región de la Orinoquia, se ha experimentado un incremento importante en la superficie forestal aprovechada en términos comerciales, generando expectativas para los territorios que cuentan con suelos con potencial forestal. A pesar de este potencial a nivel nacional y del inicio de acciones a nivel regional, en el departamento de Arauca aún no se avanza en identificar las particularidades sociales, tecnológicas, económicas y ambientales, que soporten el desarrollo de esta cadena. Esta falencia se evidencia hoy día por la ausencia de la cadena productiva forestal del departamento de Arauca, 
y de documentos que contribuyan a su conformación. En el año 2006, la Fundación para el Desarrollo Agrícola, Social y Tecnológico realizó para la Gobernación del departamento de Arauca, el Marco General y Conceptual del Sector Forestal en la Amazorinoquia, expuso de manera general las posibilidades de desarrollo del sector forestal dada la implementación del Plan Estratégico de Productividad y Competitividad del Departamento; en dicho diagnóstico se afirmaba que "no se presentan eslabones determinantes que conlleven a pensar que es posible su conformación como sistema a nivel departamental o regional a corto o mediano plazo". Posteriormente se diseñó el Plan Estratégico del Sector Forestal del Departamento de Arauca: Lineamientos para la Creación del Consejo Departamental Forestal, en el cual se ponía de manifiesto el potencial de uso del suelo asociado al desarrollo de la cadena productiva forestal; sin embargo, se evidenciaba la escasa información alrededor de la cadena; esta situación prevalece hoy día al no haber sido definidos los agentes y eslabones que deberían conformar esta cadena en el departamento, debido a la ausencia de información actualizada y la consolidación de la información existente que facilite la toma de decisiones y la definición de un plan a seguir, acorde con las necesidades y potencialidades del Departamento.

Desde el año 2006, el Departamento ha venido realizando acciones en torno a la posibilidad de iniciar el proceso de desarrollo del sector forestal; sin embargo, los resultados no han sido los esperados y, aún hoy, a pesar de haberse constituido como una de las cadenas productivas priorizadas para desarrollar en los próximos años, se observan deficiencias en la definición y coordinación de acciones que faciliten su desarrollo, tanto que se ha desaprovechado el trabajo que ha venido realizando la UPRA desde el nivel nacional, registrada en la Memoria Técnica de la Zonificación para Plantaciones Forestales con Fines Comerciales, para la orientación de acciones de planificación en las áreas rurales de Colombia, en relación con la identificación de las áreas geográficas del Departamento donde confluyen el potencial agroecológico, o áreas de vocación forestal, y el capital social disponible, requerido para que la actividad forestal se convierta en un componente estratégico en la "generación de nuevas oportunidades sociales, el fortalecimiento de la viabilidad económica territorial, el mejoramiento de la capacidad de inversión y el cuidado del medio ambiente".

Adicionalmente, la indeterminación de la estructura de los agentes que participan en la cadena productiva forestal del departamento de Arauca, hace que aún no se cuente con un plan organizado y sistemático a seguir para el desarrollo de esta cadena productiva en este territorio. La falta de organización del sector forestal en el departamento de Arauca, sumado al aprovechamiento de maderas para el consumo doméstico y el comercio ilegal, han generado la reducción y la fragmentación progresiva de los bosques naturales. El inventario de bosques para la mitad del siglo pasado era de más de un millón de hectáreas, sin embargo, según estadísticas consolidados por la gobernación del Departamento, para el año 2000, tan solo se contaba con alrededor de 220 mil hectáreas.

De acuerdo con cifras de Corporinoquia, Arauca cuenta con 137.493 has de áreas potenciales para la protección de los recursos forestales 114.680 haa para la protección-producción en zona de montaña, y 360.142 has, en zona de sabana, áreas en las que es posible aprovechar inicialmente los beneficios asociados a los sistemas forestales para luego realizar un aprovechamiento del componente maderable. Se destaca también la existencia de 446.345 has con vocación productora, en las que es posible el establecimiento de plantaciones forestales. En el departamento de Arauca las plantaciones forestales comerciales, que corresponden al tipo productor y protector productor, mencionado anteriormente:

..."pueden convertirse en una importante solución a los problemas de 
empleo de la población", pues "la vocación forestal de grandes extensiones de tierra del Departamento y la voluntad expresada por los propietarios, generan condiciones favorables para la implementación de grandes programas dirigidos al establecimiento de plantaciones forestales comerciales, así como la implementación de sistemas forestales que se articulen con los sistemas agrícolas y pecuarios tradicionales, mediante el diseño e implementación de sistemas agroforestales y silvopastoriles, que generen beneficios secundarios en torno a la conservación de los recursos naturales y el aprovechamiento sostenible de los recursos madereros".

Según el diagnóstico realizado por la Gobernación, consolidado en el Plan de Desarrollo 2012-2015, las actividades productivas y comerciales no han logrado encadenamientos que permitan a la población obtener mejores ingresos y, al Departamento, un mayor crecimiento económico y generación de empleo; para el actual período la Gobernación de Arauca planteó, dentro de sus objetivos, construir desarrollo económico enfocado en el "crecimiento sostenible y la competitividad" y, en tal sentido:

\footnotetext{
"generar un proceso de desarrollo económico endógeno a partir del mejoramiento de las capacidades de la población y del aprovechamiento de las potencialidades del territorio para que, mejorando las condiciones de productividad y competitividad, se traduzca en empleos e ingresos para los araucanos".
}

Por lo anterior se encuentra un ambiente propicio para el desarrollo de la cadena productiva forestal, con sus consecuentes aportes a la economía local, y el mejoramiento de las condiciones de empleo, principalmente en zonas rurales, para lo cual es necesario identificar y determinar la influencia de variables políticas, económicas, sociales, tecnológicas y ambientales en el futuro desarrollo de la cadena productiva forestal del Departamento de Arauca.

\section{Materiales y Métodos}

Con el fin de lograr el objetivo propuesto, la investigación fue desarrollada dos etapas. La primera etapa consistió en la identificación de los actores actuales y potenciales que conformarían la cadena productiva forestal del Departamento, para lo cual se consultó información secundaria generada por la Gobernación de Arauca, la Cámara de Comercio de Arauca y la Fundación para el Desarrollo Agrícola, Social y Tecnológico - Fundaset y Corporinoquia Seccional Arauca y la Secretaría de Desarrollo Agropecuario y Sostenible, obteniéndose la primera línea de información sobre los actores actuales y potenciales que podrían estar vinculados, a la cadena productiva forestal del departamento de Arauca. Una vez identificados los actores, se procedió a realizar el análisis PESTA correspondiente a la segunda etapa, para lo cual se realizó una consulta previa con algunos de estos actores para delinear el instrumento de recolección de información. Para esto, inicialmente, se aplicó un instrumento preliminar que contenía preguntas que debían ser calificadas por los actores, en una escala de uno (1) a cinco (5); donde uno (1) hace referencia a que no hay incidencia o impacto, dos (2) a un impacto bajo, tres (3) a un impacto mediano, cuatro (4) a un impacto alto y cinco (5) cuando no aplica. Las preguntas realizadas estaban asociadas a cada tipo de factor (político, económico, social, tecnológico y ambiental). Al finalizar las preguntas, se solicitó a los actores consultados la adición de preguntas o variables, si así lo consideraban pertinente, de acuerdo a los tipos presentados. Este ejercicio facilitó la consolidación del instrumento a aplicar. Con los actores y el instrumento definitivo de tipo encuesta, se aplicó a 42 actores, entre los cuales se encuentran profesionales de la Ingeniería Forestal que realizan actividades laborales en el Departamento, potenciales productores o dueños de tierra con interés en el sector, dirigentes del sector público relacionados con el tema agrario, transformadores de madera y comercializadores de productos terminados, resumiéndose los actores en una tabla que los compila para facilitar su análisis posterior (ver Tabla 2). 
Tabla 2: Compilado de actores de la cadena productiva forestal del departamento de Arauca consultados

\begin{tabular}{|l|l|l|}
\hline TIPO DE ACTOR & NOMBRE DE IDENTIFICACIÓN DEL ACTOR & TIPO DE INTERÉS \\
\hline Público & $\begin{array}{l}\text { Secretaría de Desarrollo Agropecuario y Sostenible del } \\
\text { departamento de Arauca }\end{array}$ & $\begin{array}{l}\text { Político, Económico, Social Tecnológico, Am- } \\
\text { biental }\end{array}$ \\
\hline Público & $\begin{array}{l}\text { Secretaría de Agricultura, Ganadería y Medio Ambiente } \\
\text { del municipio de Arauca }\end{array}$ & $\begin{array}{l}\text { Político, Económico, Social Tecnológico, Am- } \\
\text { biental }\end{array}$ \\
\hline Público & Secretaría de Planeación del municipio de Arauca & $\begin{array}{l}\text { Político, Económico, Social Tecnológico, Am- } \\
\text { biental }\end{array}$ \\
\hline Público & Alcaldía de Saravena & $\begin{array}{l}\text { Político, Económico, Social Tecnológico, Am- } \\
\text { biental }\end{array}$ \\
\hline Público & Secretaría de Agricultura del municipio de Saravena & $\begin{array}{l}\text { Político, Económico, Social, Tecnológico, Am- } \\
\text { biental }\end{array}$ \\
\hline Público & Parques Nacionales Naturales - Tame & Social, Ambiental \\
\hline Público & Corporinoquia - Seccional Arauca & Social, Tecnológico, Ambiental \\
\hline Público & ICA - Seccional Arauca & Social, Tecnológico Ambiental \\
\hline Privado & $\begin{array}{l}\text { Profesionales de Ingeniería Forestal en el departamento } \\
\text { de Arauca }\end{array}$ & Social, Tecnológico, Ambiental \\
\hline Privado & $\begin{array}{l}\text { Ex dirigencia del sector público (área de planeación } \\
\text { departamental) }\end{array}$ & Económico, Social, Ambiental \\
\hline Privado & $\begin{array}{l}\text { Dueños de tierra en el Departamento (interesados en } \\
\text { invertir en actividades forestales) }\end{array}$ & Económico, Social Ambiental. \\
\hline Privado & $\begin{array}{l}\text { Profesionales del sector agropecuario (que han trabajado } \\
\text { en el sector forestal) - Líderes del sector primario }\end{array}$ & Económico, Social Ambiental. \\
\hline Privado & $\begin{array}{l}\text { Transformadores de la madera (carpinterías) - comer- } \\
\text { cializadores de productos terminados en madera }\end{array}$ & Económico, Social Ambiental \\
\hline Privado & Sector cacaotero (sector agroforestal) & Económico, Social Ambiental \\
\hline Privado & $\begin{array}{l}\text { Ex dirigencia de Corporinoquia - seccional Arauca } \\
\text { sector forestal }\end{array}$ & $\begin{array}{l}\text { Político, Económico Social, Tecnológico, Am- } \\
\text { biental }\end{array}$ \\
\hline Privado & Económico, Social Ambiental \\
\hline
\end{tabular}

Se aplicó la encuesta a los 42 actores lo que permitió establecer 28 variables, que se considera pueden llegar a incidir en el desarrollo de la cadena productiva forestal del Departamento de Arauca, las cuales fueron organizadas por los expertos, teniendo en cuenta su impacto, calificando cada una de las variables obtenidas de cero (0) a cinco (5), de la siguiente manera: 1 - indica que hay un impacto bajo, 2 - indica que existe un impacto medio, 3 - indica la existencia de un impacto alto, 4 - indica que existe un impacto muy alto y 5 - indica que hay un impacto potencial. De esta manera se obtuvieron los códigos de las variables. Para su organización, se tomó la suma total de las calificaciones y los promedios y, para aquellas variables cuya calificación es igual, se procedió a organizar por orden alfabético. Posteriormente, se solicitó a los siete (7) expertos de la cadena, con localización en el departamento de Arauca, seleccionados por su experiencia profesional en el sector forestal y liderazgo en el desarrollo de procesos agroindustriales, seguimiento y control de planta- ciones forestales con fines comerciales y en el desarrollo de procesos de planeación a nivel del Departamento, la calificación el grado de motricidad (Influencia que una variable tiene sobre las demás variables) y dependencia (El grado en que la variable recibe influencia de todas las demás) (Ganga, 2015, Godet, 2000) de factores políticos, económicos, sociales, tecnológicos y ambientales, inicialmente por los actores, utilizando una matriz de doble entrada, facilitando la organización de las variables previamente identificadas. Luego de consolidar la información obtenida de los expertos, éstos la revisaron nuevamente y la validaron.

Finalmente, se diseñó un segundo instrumento para determinar el grado de gobernabilidad e importancia de cada variable identificada, para lo cual se presentó cada variable con su definición y se solicitó la calificación para cada una de ellas. Esta encuesta fue aplicada a 42 actores, contenía variables asociadas a los diferentes tipos de factores, las cuales debían ser cal- 
ificadas de 1 a 10 por los actores consultados, así: de 1 a $3=$ No importante o No gobernable, de 4 a $7=$ Poco importante o Poco gobernable y de 8 a $10=$ Importante o Gobernable.

En este proceso de recolección primaria se hicieron visitas de campo a los actores y, simultáneamente con el instrumento, se realizaron 13 entrevistas semi-estructuradas, lo cual permitió la recolección de información adicional y actual del sector y de la cadena productiva forestal del Departamento. En algunos casos las entrevistas fueron realizadas vía telefónica y correo electrónico, dada la dificultad de desplaza- miento hacia municipios lejanos de la capital de Arauca.

Finalmente, se obtiene el análisis PESTA, del análisis de los factores políticos, económicos, sociales, tecnológicos y ambientales (Universidad Nacional de Colombia- Sede Bogotá, 2012). Estos factores son por lo general externos y deben ser evaluados para tomar decisiones respecto de los mismos.

A continuación, se presenta un resumen de las fases en que se desarrolla la metodología (ver Tabla 3).

Tabla 3: Fases Metodológicas

\begin{tabular}{|c|c|c|c|}
\hline FASES & $\begin{array}{l}\text { INSTRUMENTOS Y/O } \\
\text { MEDIOS UTILIZADOS }\end{array}$ & $\begin{array}{l}\text { INSTITUCIONES, ENTIDADES, } \\
\text { ACTORES Y/O EXPERTOS } \\
\text { CONSULTADOS }\end{array}$ & RESULTADOS \\
\hline $\begin{array}{l}\text { Primera } \\
\text { Fase: Iden- } \\
\text { tificación de } \\
\text { actores }\end{array}$ & $\begin{array}{l}\text { Consulta de información } \\
\text { secundaria }\end{array}$ & $\begin{array}{l}\text { Cinco (5) Instituciones y entidades } \\
\text { públicas y privadas }\end{array}$ & 41 actores y 7 expertos identificados \\
\hline \multirow[t]{6}{*}{$\begin{array}{l}\text { Segunda } \\
\text { Fase: Análi- } \\
\text { sis PESTA }\end{array}$} & $\begin{array}{l}\text { Instrumento inicial para } \\
\text { recolección de informa- } \\
\text { ción para la consolidación } \\
\text { del instrumento final (val- } \\
\text { idación de instrumento). }\end{array}$ & 14 actores & $\begin{array}{l}\text { Consolidación del instrumento final a } \\
\text { aplicar }\end{array}$ \\
\hline & Instrumento final & 42 actores & $\begin{array}{l}\text { Identificación de variables que pueden } \\
\text { incidir en el desarrollo de la cadena } \\
\text { productiva forestal }\end{array}$ \\
\hline & & 7 expertos & $\begin{array}{l}\text { Codificación de las variables según su } \\
\text { impacto }\end{array}$ \\
\hline & Matriz de doble entrada & 7 expertos & Gráfico de Motricidad y dependencia \\
\hline & $\begin{array}{l}\text { Instrumento modificado } \\
\text { con variables identificadas }\end{array}$ & 42 actores 7 expertos & $\begin{array}{l}\text { Gráfico de Gobernabilidad e Importan- } \\
\text { cia }\end{array}$ \\
\hline & $\begin{array}{l}\text { Entrevista } \\
\text { estructurada }\end{array}$ & 13 actores & Información adicional \\
\hline
\end{tabular}

\section{Resultados}

En el departamento de Arauca, los recursos forestales son utilizados principalmente como fuente de maderas para la transformación, en la incipiente industria maderera local, representada por las carpinterías, las cuales actúan como comercializadores, con mueblerías locales (ver figura 1). De la misma manera, se utiliza madera del bosque a nivel doméstico, en las fincas como combustible, y como materia prima para la elaboración de artesanías, en menor proporción.
Figura 1: Comportamiento actual del sector forestal del departamento de Arauca

Imagenes/fig701.eps

Fuente: Elaboración Propia

Según información obtenida de empresas dedicadas a la transformación de la madera del Departamento, se encontró que el $29 \%$ utiliza madera de planta- 
ciones forestales comerciales y el $57 \%$ utiliza madera del bosque natural, la cual es entregada con los correspondientes permisos de aprovechamiento forestal, otorgados por la autoridad ambiental de la región (Corporinoquia, Seccional Arauca). Adicionalmente, se encontró que el origen del $14 \%$ de la madera es desconocido por los compradores, dado que realizan adquisiciones de pequeñas cantidades, de manera frecuente, a diferentes proveedores. Las maderas obtenidas se pueden clasificar en dos tipos generales: madera ordinaria y maderas finas. Las primeras, son utilizadas en la fabricación de materiales para el uso en obras civiles, tales como formaletas, tablas, tablones y listones, entre otros productos. También se fabrican postes para el cercado de fincas. Estas maderas provienen de la extracción de individuos de especies forestales como ceiba (Ceiba pentandra) e higuerón (Ficus citrifolia Mill). El segundo tipo de maderas, las denominadas maderas finas, provenientes de especies como el cedro (Cedrela odorata), amarillón (Copaifera pubiflora), roble (Platymiscium pinnatum) y ceiba tolúa (Pachira quinata), son utilizadas para la fabricación de muebles para la comercialización directa a consumidores finales y para empresas pequeñas dedicadas a la comercialización de muebles. Los volúmenes adquiridos anual- mente varían de acuerdo con el tamaño de la empresa, realizándose adquisiciones entre 40 y $1500 \mathrm{~m} 3$, obtenidas a través de uno o dos proveedores, quienes establecen el precio de la madera y con los cuales se mantiene una relación comercial que se rige por la antigüedad en la relación $y$, en tal sentido, por la confianza proveedorcomprador. El origen de las maderas utilizadas por los transformadores de la cadena forestal en el departamento de Arauca corresponden a las obtenidas del departamento de Norte de Santander, especialmente de los municipios de Cúcuta y Pamplona, de donde proviene la mayor cantidad (67\% del total); aunque también se obtienen maderas procedentes de diferentes zonas del departamento de Arauca (33\%), mientras que el $14 \%$ proviene de otros lugares.

\section{Análisis estratégico de la cadena productiva forestal del departamento de Arauca}

De la aplicación del segundo instrumento (encuesta), se obtuvo una lista de 28 variables, las cuales fueron clasificadas como de tipo político, económico, social, tecnológico y ambiental (ver Tabla 4). Tabla 4. Variables de tipo político, económico, social, tecnológico y ambiental, identificadas por actores del sector forestal en el departamento de Arauca.

Tabla 4

\begin{tabular}{|c|c|c|}
\hline FACTORES & VARIABLES & DESCRIPCIÓN \\
\hline \multirow[t]{4}{*}{ POLÍTICOS } & Conflicto armado & $\begin{array}{l}\text { Presencia de grupos armados al margen de la ley en las zonas con potencial } \\
\text { para el desarrollo de la actividad forestal que reduce las posibles inversiones en } \\
\text { el sector, en las zonas rurales de los municipios del departamento de Arauca y } \\
\text { dificulta las actividades logísticas y de comercialización de la cadena productiva. }\end{array}$ \\
\hline & Visión departamental & $\begin{array}{l}\text { Visión del gobierno departamental actual y de los próximos períodos admin- } \\
\text { istrativos, en relación con el desarrollo de la cadena productiva forestal de } \\
\text { Arauca. }\end{array}$ \\
\hline & $\begin{array}{l}\text { Normas de control ambiental y } \\
\text { forestal }\end{array}$ & $\begin{array}{l}\text { Normas vigentes y expedición de normatividad nueva, tanto nacional como } \\
\text { regional, que puede estar en contravía del desarrollo de la cadena productiva } \\
\text { forestal de Arauca, generando restricciones legales para el desarrollo de la } \\
\text { cadena y sus diferentes eslabones. }\end{array}$ \\
\hline & Políticas nacionales & $\begin{array}{l}\text { Existencia del Plan Nacional de Desarrollo Forestal y otras políticas nacionales } \\
\text { y su incidencia en el desarrollo de la cadena productiva forestal en el } \\
\text { departamento de Arauca. }\end{array}$ \\
\hline \multirow[t]{4}{*}{ ECONÓMICOS } & Inversión privada & $\begin{array}{l}\text { Invertir capitales privados tanto en el establecimiento de plantaciones forestales } \\
\text { comerciales (producción forestal), como en actividades de transformación } \\
\text { (aprovechamiento forestal) y comercialización de productos terminados. }\end{array}$ \\
\hline & Inversión pública & $\begin{array}{l}\text { Presencia y articulación institucional de las entidades territoriales y nacionales } \\
\text { para mantener líneas de inversión en todas las fases de proyectos forestales } \\
\text { comerciales y, en tal sentido, a favor del desarrollo de la cadena productiva } \\
\text { forestal. }\end{array}$ \\
\hline & Insumos y otros & $\begin{array}{l}\text { Costo de los insumos, materiales, herramientas y elementos para el desarrollo } \\
\text { de trabajos en plantaciones forestales y las actividades de aprovechamiento de } \\
\text { productos y servicios ambientales. }\end{array}$ \\
\hline & Mano de obra & $\begin{array}{l}\text { Costo de la mano de obra acorde con el contexto, con las necesidades de los } \\
\text { pobladores de las zonas rurales y con las necesidades de desarrollo de la cadena } \\
\text { productiva forestal, promoviendo al mismo tiempo su disponibilidad. }\end{array}$ \\
\hline
\end{tabular}




\begin{tabular}{|c|c|c|}
\hline FACTORES & VARIABLES & DESCRIPCIÓN \\
\hline & Tenencia de la tierra & $\begin{array}{l}\text { Participación de los dueños de la tierra en el departamento de Arauca, } \\
\text { en proyectos forestales comerciales, asociados al desarrollo de la cadena } \\
\text { productiva. }\end{array}$ \\
\hline & Ubicación geográfica de áreas & $\begin{array}{l}\text { Las áreas con potencial para el establecimiento de plantaciones forestales } \\
\text { comerciales se encuentran en zonas de difícil acceso. }\end{array}$ \\
\hline & Competencia desleal & $\begin{array}{l}\text { Participación igualitaria en el mercado de productos y servicios forestales entre } \\
\text { aprovechadores del bosque natural y quienes realizan plantaciones forestales } \\
\text { comerciales, con costos desde el establecimiento hasta el aprovechamiento. }\end{array}$ \\
\hline & Identificación de mercados & $\begin{array}{l}\text { Identificación de mercados para los productos y servicios generados a través de } \\
\text { la cadena productiva forestal del departamento de Arauca. }\end{array}$ \\
\hline & Tasa de empleo rural & $\begin{array}{l}\text { Aumento de la tasa de empleo rural con el desarrollo de la cadena productiva } \\
\text { forestal. }\end{array}$ \\
\hline \multirow[t]{7}{*}{ SOCIALES } & Desconocimiento del sector & $\begin{array}{l}\text { Reducción del desconocimiento de los actores de la cadena productiva forestal } \\
\text { sobre el potencial de aprovechamiento económico de productos y servicios } \\
\text { ambientales del bosque, además de los incentivos, programas de financiación y } \\
\text { apoyos gubernamentales para el desarrollo del sector forestal. }\end{array}$ \\
\hline & Asociatividad & $\begin{array}{l}\text { Desarrollo de procesos de asociatividad e integración entre productores } \\
\text { y potenciales transformadores y comercializadores, dejando de lado el } \\
\text { individualismo. }\end{array}$ \\
\hline & Tendencia productiva tradicional & $\begin{array}{l}\text { Desarrollo de procesos de producción diferentes a la ganadería y la agricultura, } \\
\text { tradicionales en la región. }\end{array}$ \\
\hline & $\begin{array}{l}\text { Educación técnica y superior } \\
\text { para el sector }\end{array}$ & $\begin{array}{l}\text { Desarrollo de procesos de formación académica, a nivel técnico, tecnológico } \\
\text { y superior, en las ciencias forestales y, en general, en programas académicos } \\
\text { requeridos para el desarrollo de la cadena, enfocados en la cualificación de las } \\
\text { personas del departamento de Arauca. }\end{array}$ \\
\hline & Informalidad & $\begin{array}{l}\text { Creación de empresa en el departamento de Arauca, relacionadas con la cadena } \\
\text { productiva forestal. }\end{array}$ \\
\hline & $\begin{array}{l}\text { Percepción de riesgo en la inver- } \\
\text { sión }\end{array}$ & $\begin{array}{l}\text { Disminución de la percepción del riesgo en la inversión de recursos en proyectos } \\
\text { de largo plazo (tal como los forestales), tanto en los dueños de la tierra como } \\
\text { en las personas del departamento de Arauca. }\end{array}$ \\
\hline & Nivel socioeconómico & $\begin{array}{l}\text { Mejoramiento del nivel socio-económico de las comunidades rurales con el } \\
\text { desarrollo de la cadena productiva forestal. }\end{array}$ \\
\hline \multirow[t]{4}{*}{ TECNOLÓGICOS } & Asistencia técnica & $\begin{array}{l}\text { Acceso a la asistencia técnica por parte de productores de bienes y servicios } \\
\text { forestales, mediante el establecimiento de costos acordes con el contexto y con } \\
\text { las necesidades de desarrollo de la cadena productiva forestal. }\end{array}$ \\
\hline & Disponibilidad de maquinaria & $\begin{array}{l}\text { Acceso a la maquinaria especializada para el establecimiento, mantenimiento } \\
\text { y aprovechamiento de plantaciones forestales comerciales, así como para la } \\
\text { transformación de productos y la generación de valor agregado, a costos acordes } \\
\text { a las características y condiciones del departamento de Arauca. }\end{array}$ \\
\hline & Desarrollo tecnológico & $\begin{array}{l}\text { Acceso a paquetes tecnológicos del sector forestal a los productores de la cadena } \\
\text { en el departamento de Arauca. Desarrollo de procesos de generación de valor } \\
\text { agregado basado en el desarrollo tecnológico. }\end{array}$ \\
\hline & Investigación & $\begin{array}{l}\text { Desarrollo de la investigación mediante la asignación de recursos que faciliten la } \\
\text { determinación de especies forestales con mayor potencial de aprovechamiento, } \\
\text { incluyendo especies forestales nativas con alto potencial maderero, así como } \\
\text { la creación de valor agregado y el desarrollo de procesos de comercialización } \\
\text { basado en investigación de mercados, mejorando prácticas. }\end{array}$ \\
\hline \multirow[t]{4}{*}{ AMBIENTALES } & Conservación ambiental & $\begin{array}{l}\text { Desarrollo de procesos de establecimiento y aprovechamiento con prácticas que } \\
\text { no generen impactos negativos sobre el ambiente (suelos, agua subterránea y } \\
\text { superficial, fauna asociada y flora circundante). }\end{array}$ \\
\hline & Bosques naturales & $\begin{array}{l}\text { Regeneración y la conservación de bosques naturales, mediante el desarrollo de } \\
\text { plantaciones forestales comerciales. }\end{array}$ \\
\hline & Sensibilidad ambiental & $\begin{array}{l}\text { Promoción de la sensibilidad ambiental en los actores de la cadena productiva, } \\
\text { para evitar el deterioro de las áreas en las cuales se realizan proyectos forestales. }\end{array}$ \\
\hline & Vocación del suelo & $\begin{array}{l}\text { Definición clara de la totalidad de las áreas con vocación forestal en el } \\
\text { departamento de Arauca y su socialización en la comunidad. }\end{array}$ \\
\hline
\end{tabular}

A continuación, se presenta el listado de variables, producto de la calificación por expertos, clasificadas de acuerdo con su impacto, teniendo presente que el código 1 corresponde a la variable de mayor impacto y, el código 28, a la variable de menor impacto (ver figura 2).

Para la realización de este análisis, se tuvo en cuenta el resultado de las entrevistas a los actores de la cadena productiva forestal del departamento de Arauca, con el fin de identificar los problemas que podrían afectar su desarrollo. El análi- sis PESTA del sector forestal (ver figura 2) muestra la preponderancia de variables económicas y sociales, las cuales representan el $35.71 \%$ y el $21.43 \%$ de los factores analizados. Dada la calificación obtenida de los actores de la cadena, se observa una fuerte incidencia de la variable conflicto armado (1), de los factores políticos, considerado de gran impacto en el desarrollo de la cadena, en este primer ejercicio, que refleja el efecto de la presencia de grupos armados al margen de la ley en el Departamento. 
Figura 2: Análisis PESTA del sector forestal del departamento de Arauca

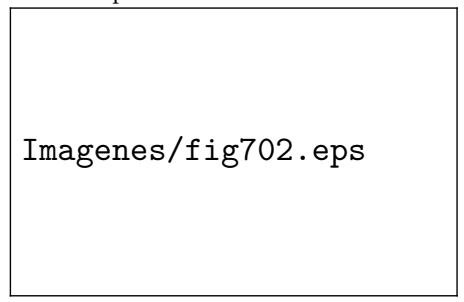

Fuente: Elaboración Propia

Se identificaron las variables clave, para lo cual fue necesario realizar el análisis estructura a partir de los resultados obtenidos en el análisis PESTA, para ello se determinó la incidencia y la importancia de cada variable sobre las demás. El análisis estructural se constituye como un "elemento de análisis" que "hace parte de los métodos prospectivos y facilita la interpretación de la realidad, concibiéndola como un sistema, cuyas partes no están sueltas, sino en estrecha relación, de tal forma que un cambio que se presente en uno de sus elementos, repercute directa o indirectamente en los restantes" (Montoya et al, 2005, pág. 108). De hecho, "el objetivo del análisis estructural es precisamente poner de relieve la 'estructura' de las relaciones entre las variables cualitativas, cuantificables o no, que caracterizan el sistema estudiado" y reduciendo el entramado complejo de variables a las esenciales. Utilizando una tabla de doble entrada, se organizaron las variables en columnas y en filas, de tal manera que al cruzarlas se pudieran obtener las relaciones existentes entre ellas. Teniendo esta matriz, se solicitó nuevamente al grupo de expertos que indicaran si cada variable ordenada en la columna influía, de manera directa o potencial, sobre cada una de las variables ordenadas en la fila, utilizando para ello la misma escala de calificación utilizada anteriormente:

Una vez consultados a los siete (7) expertos, se usaron las matrices individuales calificadas por los expertos, y se procedió a unificar los resultados en una sola matriz para la cadena forestal usando para ello la media aritmética para alimentar cada una de las celdas de la matriz grupal. Seguidamente, se procedió a realizar la sumatoria de las columnas y de las filas. Todas las cifras se tradujeron a términos porcentuales. El resultado de la sumatoria de las filas indica el número de veces que cada variable impacta o influye directamente a las demás, el cual se constituye en el índice de motricidad. De la misma manera, se trabajaron las columnas, cuyo resultado porcentual indica las veces en que cada variable depende de las demás; es decir, el índice de dependencia de cada variable. En consecuencia, se obtuvo una matriz unificada con los porcentajes de motricidad y dependencia para la cadena productiva forestal del departamento de Arauca, a partir de la cual se generó un gráfico que muestra los diferentes puntos que comportan tanto el índice de dependencia (eje X) como el índice de motricidad (eje Y), de un plano cartesiano (ver figura 3).Cada uno de los números del gráfico corresponde al código asignado a las variables identificadas, previamente presentadas en la tabla 4 .

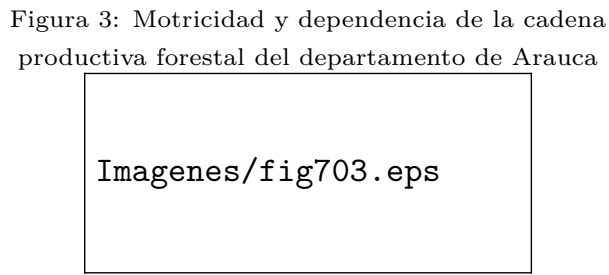

De la figura 3 se obtiene que las variables localizadas en la zona de poder, es decir que ejercen una fuerte influencia sobre las demás variables pero, simultáneamente, permiten poca influencia por las mismas,son: educación técnica y superior para el sector (12), vocación del suelo (15), ubicación geográfica de áreas (20), competencia desleal (22) y desconocimiento del sector (27),

En las entrevistas realizadas a los actores de la cadena, fue resaltada la importancia de contar con personal calificado y capacitado para el sector forestal (12), ev- 
idenciada a través de la escasa oferta de profesionales del área en el departamento de Arauca y la permanencia tan solo de cinco (5) en la región, aunque ninguno de ellos trabajando en actividades productivas del sector forestal. De la misma manera, los entrevistados manifestaron que, para el desarrollo de procesos operativos de producción de bienes y servicios ambientales, así como de transformación de éstos en productos comercializables, se requiere personal del nivel técnico y tecnológico al servicio de los proyectos del sector.

El desconocimiento del sector forestal (27), presenta un índice de motricidad que está por encima del $78.57 \%$ de las variables $(5.96 \%)$ y una dependencia baja $(2.74 \%)$, si se compara su posición con respecto a las demás variables (se encuentra sobre el $25 \%$ de ellas), según manifestaron los entrevistados, ha generado el bajo interés de potenciales productores de bienes y servicios del bosque en la inversión de recursos por parte de los dueños de tierra en el Departamento, así como de potenciales inversionistas, ocasionando además un efecto colateral relacionado con la percepción del riesgo en la inversión de recursos privados y públicos, la preferencia de actividades productivas tradicionales sobre la producción forestal y la imposibilidad de identificar mercados potenciales para productos y servicios forestales generados en el Departamento, lo que a su vez repercute indirectamente en el nivel socioeconómico y la tasa de empleo en las zonas rurales, el desarrollo de procesos de comercialización bajo un manto de informalidad y la degradación de áreas boscosas naturales.

La competencia desleal (22), presenta un índice de motricidad de $4.05 \%$, lo que indica que está por encima del $67.86 \%$ de las variables, y un índice de dependencia del $1.79 \%$ (por encima del $14.29 \%$ de las variables); lo que está directamente relacionado con la desigualdad generada por la presencia en el mercado de comercializadores de productos forestales provenientes plantaciones comerciales, los cuales han realizado la inversión de recursos desde la fase de establecimiento hasta la fase de aprovechamiento, frente a la presencia de aprovechadores del bosque natural, que han realizado una escasa inversión, pero que acceden al mercado con las mismas posibilidades.

Tanto la variable vocación del suelo (15) como ubicación geográfica de áreas (20), tienen una dependencia baja (índice de dependencia del $0.45 \%$ y $0.51 \%$, respectivamente) de las demás variables, mientras que presentan un índice de motricidad superior al del $60.71 \%$ de las variables identificadas por los actores de la cadena ( $4.03 \%$ y $4.05 \%$, respectivamente), lo que indica que, además de comportar por sí mismas la relevancia dada por la dificultad de acceder a zonas con vocación forestal, debido a la presencia de grupos armados al margen de la ley y a las condiciones deficientes de las vías de acceso, influyen fuertemente sobre otras variables como la inversión pública y la inversión privada.

La variable ubicación geográfica de áreas presenta un índice de motricidad del $3.63 \%$, superior al del $53.57 \%$ de las variables identificadas en este estudio, lo que muestra el grado de influencia ejercido sobre las demás variables, dado que por ejemplo los terrenos con vocación forestal se encuentran en zonas de difícil acceso, donde hay presencia de grupos armados al margen de la ley y las condiciones de las vías de acceso son deficientes.

Las variables encontradas en la zona de conflicto representan problemas relacionados con la inversión de recursos, tanto públicos (3) como privados (18), en la visión departamental relacionada con la cadena (21), en el desarrollo de procesos de investigación (10), en la superación de la informalidad de los actores de la cadena (13), en el desarrollo de procesos de asociatividad de actores (2), en el desarrollo tecnológico (7), en el nivel socioeconómico (25) fundamentalmente de las comunidades rurales y en la asistencia técnica asociada al desarrollo de la cadena productiva forestal (25) (ver figura 5). En 
consecuencia, la presencia de estas variables en la zona de conflicto permite prever la necesidad de un trabajo conjunto entre los actores de los diferentes eslabones de la cadena productiva, gestionando variables que, a su vez, permitan influir positivamente en las demás.

Las variables que presentan mayor motricidad en esta zona son: inversión privada (18), visión departamental (21) e inversión pública (3), con índices de motricidad de $8.00 \%, 7.39 \%$ y $7.11 \%$, respectivamente; indicando una fuerte influencia sobre las demás variables identificadas para la cadena. De la misma manera, los mayores índices de dependencia los presentan estas mismas variables, donde la inversión pública presenta un $6.78 \%$ de dependencia; inversión privada, con $6.48 \%$ y visión departamental, con $5.48 \%$ de dependencia, todas ellas con un índice de dependencia superior del $89.29 \%$ de las variables. En la zona de problemas (ver Tabla 5), se encuentran las variables: conflicto armado (1), disponibilidad de maquinaria (8), insumos y otros (9), identificación de mercados (11), mano de obra (14), tenencia de la tierra (17), normas de control ambiental y forestal (23) y políticas nacionales (28).

En la zona de salida, se encuentran las variables: sensibilidad ambiental (4), bosques naturales (5), conservación ambiental (6), percepción de riesgo en la inversión (16), tendencia productiva tradicional (19) y tasa de empleo rural (26)

Teniendo identificada la posición de cada de una de las variables asociadas a la cadena productiva forestal del departamento de Arauca, en los cuadrantes, es importante trabajar en las variables localizadas en la zona de poder, debido a que su efecto incidirá en las variables localizadas en las zonas restantes. Seguidamente, en las variables localizadas en la zona de conflicto debido a su función de enlace entre la zona de poder y las zonas de problemas y salida. Se solicitó además a los actores de la cadena productiva forestal identificados, incluyendo los expertos, que realizaran la calificación de los factores en relación con el grado de importancia y de gobernabilidad o manejo de cada uno de ellos para el futuro de la cadena productiva (de 1 a 10). En este caso, se tomó una escala más amplia para facilitar la lectura de la gráfica, luego de la inclusión de los resultados. Obtenida la calificación, se realizó la unificación de los resultados mediante la obtención de promedios estadísticos de las respuestas, los cuales fueron llevados a un plano cartesiano para su representación (ver figura 4).

Figura 4: Gobernabilidad e importancia de la cadena productiva forestal del departamento de Arauca, realizada con la información obtenida de los actores de la cadena

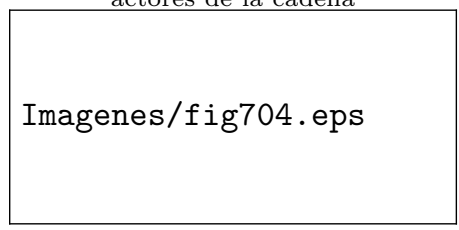

Fuente: Elaboración Propia

\section{Discusión}

Una vez se contrastaron estos resultados con los obtenidos en el análisis estructural, se estableció que las variables tenían los siguientes comportamientos:

- La variable conflicto armado (1), que en el análisis estructural se encontraba en la zona de problemas, en el análisis, aún se mantiene en el mismo cuadrante, indicando una muy baja gobernabilidad (3.59), a pesar de su importancia (8.12), aunque esta se encuentra por debajo del promedio.

- Las variables asociatividad (2), inversión pública (3) y desarrollo tecnológico (7), que en el análisis estructural se encontraban en la zona de conflicto, también se mantienen en el mismo cuadrante, indicando que hay posibilidades de manejo, dada su importancia para el desarrollo de la cadena productiva (9.17). 
- Las variables sensibilidad ambiental (4), bosques naturales (5) y conservación ambiental (6), a pesar de mostrarse dependientes, se presentan ahora como importantes $(9.27 ; 9.07 ; 9.02$; respectivamente) y manejables $(5.76 ; 5.71 \mathrm{y}$ 6.20 ; respectivamente), y esto puede estar ligado al auge que ha tenido la tendencia hacia el surgimiento $\mathrm{y} / \mathrm{o}$ el fortalecimiento de una economía verde en el país.

- Los actores de la cadena consideran que, a pesar de ser dependiente de otras variables, la disponibilidad de maquinaria (8), se puede manejar $(5,68)$, debido a la necesidad que se tiene de esta, sobre todo en los procesos de transformación, razón por la cual se considera muy importante (9.10).

- La variable insumos y otros (9), se encuentra localizada en la zona de montón, el mismo cuadrante en el que se encontró en el análisis estructural. Se percibe con un nivel de importancia alto (8.41), aunque por debajo del promedio, mientras que se considera poco gobernable (4.88).

- En cuanto a la investigación (10), se considera que tiene una alta dependencia de otras variables y, al mismo tiempo, que es muy importante (9.44) y, además, de fácil manejo para la cadena productiva forestal del Departamento (6.88). Es una de las variables más importantes, tan solo por debajo de la variable educación técnica y superior para el sector (12), en nivel de importancia.

- La variable identificación de mercados (11), en el análisis estructural se mostraba como poco motriz y poco dependiente, se considera que tiene una alta importancia (8.85) y que resultaría manejable o gobernable para la cadena productiva forestal del departamento de Arauca. Esta variable presenta el mayor índice de gobernabilidad de todas las variables identificadas (7.37).
- La educación técnica y superior para el sector (12), se presenta en el análisis estructural como una variable que tiene una influencia fuerte sobre otras, así como una baja dependencia y, adicionalmente, se presenta como una variable importante para el desarrollo de la cadena (9.46) y con facilidad para su manejo (6.54), dado su nivel de gobernabilidad, la cual dependería básicamente de inversión e interés de las instituciones públicas $\mathrm{y} / \mathrm{o}$ privadas para su desarrollo.

- La informalidad (13) se encontraba en la zona de conflicto y se mantiene como una variable estratégica, muy importante (9.00) y con cierto grado de manejo (5.71).

- La variable mano de obra (14), se encuentra en la zona de problemas, con muy bajos índices de motricidad y dependencia y, a pesar de que se considera de importancia para el sector (8.24), tiene un nivel de gobernabilidad intermedio (5.71).

- La variable vocación del suelo (15), que en las entrevistas se manifestó como una variable que podría tener un nivel de importancia superior, y en el análisis estructural se mostró como una variable que hacía parte de la zona de poder, con una muy baja capacidad de influir sobre otras variables y con una muy baja dependencia de otras variables; ahora se muestra como una variable localizada en la zona de estratégicos con una alta importancia (9.39) y con posibilidades de manejo (7.00), si se compara con las demás variables identificadas.

- La percepción del riesgo en la inversión (16), se presentó en la zona de salida como una variable poco influyente sobre otras y con una dependencia intermedia, ahora se manifiesta como una variable muy importante (8.83), a pesar de ser medianamente manejable por la cadena productiva (5.41).

- La tenencia de la tierra (17) es una variable que poco influye sobre otras; sin 
embargo, se percibe ahora como muy importante (9.02), aunque poco gobernable (5.17).

- La variable inversión privada (18), a pesar de haberse identificado como de alta motricidad y alta dependencia, se confirma ahora que es de difícil manejo, pues se encuentra ubicada en la zona de montón, donde a pesar de considerarse importante (8.41), también se considera poco gobernable (4.66).

- La tendencia productiva tradicional (19) se presenta como una variable importante $(8.20)$ y medianamente gobernable (4.90), posiblemente en razón a que los actores consideran, según fue manifestado durante el desarrollo de las entrevistas, que no es necesario cambiar radicalmente el esquema productivo tradicional (ganadería y agricultura), sino que es necesario combinar esos esquemas con otros.

- La variable ubicación geográfica de áreas (20), se mantiene en el cuadrante inferior izquierdo (zona de montón), debido a que a pesar de ser importante (7.63), es de difícil manejo para la cadena (4.46).

- La visión departamental (21), se percibe como una variable, a pesar de que se considera como altamente influyente sobre otras variables y de gran importancia (8.15), se considera también de muy baja gobernabilidad (5.15), lo que podría indicar también que la entidad territorial del orden departamental no cuenta con la confianza de los actores de la cadena.

- La competencia desleal (22), a pesar de haberse localizado en la zona de poder, en el análisis estructural, ahora se presenta en la zona de montón, indicando una baja gobernabilidad (4.49) y una importancia (8.10) que, a pesar de ser alta, se encuentra por debajo del promedio (8.74).

- La variable normas de control ambiental (23) se encuentra en el cuadrante inferior izquierdo, en la zona de montón, el mismo cuadrante resultante del análisis estructural, debido a que, tanto su importancia (7.68), como su gobernabilidad (5.10), se encuentran por debajo de sus respectivos promedios (8.74 y 5.64; respectivamente).

- Tanto la asistencia técnica (24), como el nivel socioeconómico (25) se manifiestan como variables de gran importancia para el desarrollo de la cadena (9.12 y 9.00 ; respectivamente) y medianamente gobernables (6.27 y 5.78 ; respectivamente), por encima del promedio, localizándose dentro de las variables estratégicas para la cadena productiva objeto del análisis.

- La tasa de empleo rural (26), que en el análisis estructural se encontraba en la zona de salida, aquí se presenta en la zona de variables estratégicas, con una importancia superior al promedio (8.88) y una gobernabilidad también superior al promedio (5.66), indicando que los actores de la cadena consideran que al desarrollar los diferentes eslabones, se aumenta la oferta de empleo en las zonas rurales.

- El desconocimiento del sector (27), en el análisis estructural se presentaba en la zona de poder, ahora se presenta en la zona de variables estratégicas, demostrando una alta importancia para los actores de la cadena (9.20) el hecho de que se acceda a la información relacionada con el sector forestal, en los diferentes eslabones, lo cual sería manejable (6.02) por la cadena productiva.

- Las variable políticas nacionales (28), en el análisis estructural se encontraba en el cuadrante inferior izquierdo, se mantiene, localizándose en la zona de problemas, con una importancia y una gobernabilidad, por debajo del promedio (5.34 y 8.10; respectivamente).

De las variables que en el análisis estructural se encontraban en las zonas de poder y de conflicto, tan solo las variables desarrollo tecnológico (7), investigación (10), informalidad (13), asisten- 
cia técnica (24), nivel socioeconómico (25) e inversión pública (3), localizadas en la zona de conflicto, presentan posibilidades de manejo por los actores de la cadena (ver figura 5).

Figura 5: Variables de trabajo producto de la intersección entre los resultados del análisis de motricidad y dependencia y del análisis de gobernabilidad e importancia

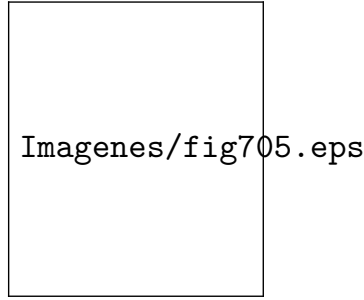

Fuente: Elaboración Propia

\section{Conclusiones}

El desarrollo de la cadena productiva forestal del departamento de Arauca se percibe aún incipiente y desagregado. De hecho, existen pocos actores y empresas trabajando alrededor de esta, todos ellos trabajando individualmente por sus objetivos sin tener en cuenta las posibilidades que ofrece la integración empresarial.

Se ha dado prioridad a la comercialización de un único producto forestal (madera), dejando de lado el potencial que tiene la comercialización y transformación de productos del bosque, tales como fibras, semillas, sustancias químicas medicinales, colorantes, aceites esenciales, gomas, maderas en pie, frutas y frutos secos, carne de caza e insectos, entre otras; y la venta de servicios ambientales y de otros productos del bosque (bonos de carbono, áreas reforestadas o forestadas, zonas de biodiversidad y turismo, entre otros), en los mercados nacionales e internacionales, los cuales pueden ser una alternativa interesante a desarrollar en la cadena forestal del departamento de Arauca.

Las materias primas obtenidas para el desarrollo de procesos de transforma- ción en el departamento de Arauca son obtenidas, en su mayoría, de otros departamentos cercanos, y las obtenidas en el territorio provienen de bosques naturales.

La inversión privada se ve reducida debido a la falta de garantías de seguridad, ocasionadas por las condiciones de orden público que vive el Departamento, especialmente en las áreas rurales, donde se encuentran tierras aptas para el establecimiento de cultivos forestales asociados a dicha cadena productiva, pero donde los factores de riesgo son también altos.

A pesar del enorme potencial forestal que ofrece el territorio del departamento de Arauca, el análisis interno y externo del sector evidencia una ausencia de claridad en la forma como se debe operacionalizar la priorización dada a la cadena productiva forestal $\mathrm{y}$, en ese sentido, se observan acciones aisladas, tanto del Gobierno local, como de particulares, que no ven el producto de sus esfuerzos técnicos, económicos y administrativos, conduciendo al deterioro de la confianza en el futuro promisorio de la cadena.

Se encuentran falencias en el sistema crediticio nacional, generadas por la ausencia de instrumentos financieros que faciliten verdaderamente el desarrollo de la actividad silvícola en el departamento de Arauca, dado que no se encuentran disponibles condiciones de crédito que correspondan con los períodos productivos de una plantación forestal comercial, cuyas tasas de interés resultan ser poco atractivas, reduciendo la inversión en este sector.

Los incentivos forestales generados por las instituciones del Gobierno Nacional, a través de programas nacionales, no corresponden con la realidad del Departamento, dado que los requisitos solicitados para su otorgamiento no son de fácil cumplimiento por los potenciales interesados en la producción forestal, reduciendo aún más las posibilidades de inversión y de acción en el sector. Es así como se ha minimizado la importancia y el impacto que podría 
tener la actividad forestal comercial en la generación de empleo rural y urbano, debido a que este tipo de cultivos podría generar más puestos de trabajo, calificado y no calificado, para la población del Departamento que hoy depende en su mayoría del sector oficial y de la actividad petrolera. Aunque al inicio de la investigación las variables asociadas a factores ambientales fueron consideradas por los actores como importantes, en análisis subsecuentes fueron quedando en un segundo plano, dando prioridad a otras variables, posiblemente porque es una cadena que aún no existe y se requiere actuar inicialmente en torno a las variables de trabajo identificadas por los actores y expertos de la cadena productiva forestal del departamento de Arauca. La integración empresarial es de suma importancia para el desarrollo de la cadena productiva forestal del departamento de Arauca debido a que, a través de la integración, coordinación y cooperación, se crean fortalezas conjuntas y sinergias en una cadena productiva incipiente como la forestal, facilitando su desarrollo e ingreso de productos y servicios a los mercados nacionales e internacionales.

\section{Referencias}

Aithal, P. S., Shailashree, V. T., \& Kumar, P. S. (2015). A new ABCD technique to analyze business models \& concepts. International Journal of Management, IT and Engineering, 5(4), 409. Disponible en: http://search.proquest.com/openview/ dcbf34b7d66c90b26ed8eafb9c6e8f8c/1?pqorigsite $=$ gscholar. Consultado 25/05/2015

Corporinoquia \& Carbono y Bosques. (2008). Plan general de ordenamiento forestal. Contrato No. 100.14.08.039 de 2008.

Departamento Nacional de Planeación. (2001). Conpes 3125: Estrategia para la consolidación del plan nacional de desarrollo forestal. Bogotá D.C. Disponible en: https://www.siac.gov.co/documentos/ DOC_Portal/DOC_Bosques/Plan_nacio nal_desarrollo forestal.pdf De la anticipación a la acción

FAO. (2007). Situación de los bosques del mundo. Disponible en:

http://www.fao.org/docrep/009/a0773s/ a0773s00.HTM De la anticipación a la acción

FAO. (2011). Situación de los bosques del mundo. Roma, Italia.

http://www.fao.org/docrep/013/i2000s/ i2000s.pdf. De la anticipación a la acción

FAO. (2014). Situación de los bosques del mundo. Roma, Italia.

http://www.fao.org/forestry/sofo/es/ De la anticipación a la acción

FUNDACIÓN PARA EL DESARROLLO AGRÍCOLA, SOCIAL Y TECNOLÓGICO - FUNDASET. (2006). Marco General y Conceptual del Sector Forestal en la Amazorinoquia. Arauca, Arauca.

FUNDASET. (2007). Plan Estratégico del Sector Forestal del Departamento de Arauca: Lineamientos para la Creación del Consejo Forestal Departamental. Arauca, Arauca.

Ganga, L. E. C. (2015). Categorías de análsis de un modelo de planificación turística sustentable. Cuadernos de Investigación Urbanística, (95). Disponible en: http://polired.upm.es/index.php/ciur/ article/view/3009/3068. Consultado $25 / 05 / 2015$

Gobernación de Arauca. (2012). Plan de Desarrollo Departamental de Arauca 2012-2015. Es Hora de Resultados. Arauca, Arauca. http://www.arauca.gov.co/documentos/ ORDENANZA\%20001E\%20PLAN DE DESARROLLO_DEPARTAMENTAL 2012-2015\%20OK.pdf. Consultado 25-052015

Godet, M. (1996). De la anticipación a la acción. Santafé de Bogotá: Alfomega S.A.

Godet, M. (2000). La caja de herramientas de la prospectiva estratégica. Gerpa. Disponible en: http://metodologiasdelainvestigacion. blog.com/files/2013/09/METODOS-DELA-PROSPECTIVA-ESTRATEGICA.pdf, Consultado 25/05/2015

https: //www.google.com.co/url?sa=t\&rct $=\mathrm{j} \& \mathrm{q}=\& \mathrm{esrc}=\mathrm{s} \&$ source $=$ web\& $\mathrm{cd}=1 \& \mathrm{cad}$ $=$ rja\&uact $=8 \&$ ved $=0 \mathrm{CBsQFjAA} \& u r l=$ http $\% 3 \mathrm{~A} \% 2 \mathrm{~F} \% 2 \mathrm{Fwww}$.territorioscentro americanos.org\% 2 Fexperiencias $\% 2$ FDocuments $\% 2$ FPlanificaci $\% 25 \mathrm{C} 3$ $\% 25$ B3n $\% 2520 y \% 2520$ Gesti\%25C3\% 25B3n\%2520de\%2520Territorios.pdf\&ei $=16 \mathrm{dj}$ VbjTDMnmsATlloDQBA\&usg $=\mathrm{AF}$ QjCNFfyeZcDrSYlYxphcW8FVUk0dRTw A\&sig2=u0qcaAHB2nrW_eklBpnr6A\&b vm $=$ bv.93990622,d.cWc. Consultado 2505-2015

Matta, J., \& Schweitzer Meins, L. (2012). Un nuevo lugar para situar los bosques dentro del sector desarrollo. FAO. Disponible en: http://www.fao.org/3/ai2890s/i2890s02.pdf. Consultado $25 / 05 / 2015$

Ministerio de Agricultura y Desarrollo $\mathrm{Ru}$ ral - UPRA. (2014). Zonificación para plantaciones forestales comerciales Escala 1:100.000. Disponible en: 
http://www.upra.gov.co/documents/1018 4/13821/ZONIFICACI\%C3\%93N+PARA +PLANTACIONES+FORESTALES+ $\mathrm{CON}+\mathrm{FINES}+\mathrm{COMERCIALES}+1-$ 100.000/8c36a1fd-a6a7-4082-89d9e9c06da74bcf. Consultado 25/05/2015

Ministerio de Agricultura y Desarrollo Rural. (2005). La cadena forestal y madera en Colombia. Bogotá. Disponible en: http://fcf.unse.edu.ar/archivos/posgrado/ 2005.salazar.cadenamadera.colombia.cen trodoc_1140.pdf Consultado 25/05/2015

Ministerio de Agricultura y Desarrollo Rural. (2007). Cadena productiva forestal - Tableros aglomerados y contrachapados Muebles y productos de madera. Bogotá, Colombia: Giro Editores Ltda. Disponible en: http://www.agronet.gov.co/www/ docs_agronet/2008313114521_forestal.pdf Consultado 25/05/2015

Ministerio del Medio Ambiente y otros. (2000). Plan nacional de desarrollo forestal. Bogotá D.C. https://www.siac.gov.co/docu mentos/DOC_Portal/DOC_Bosques/ Plan_nacional_desarrollo_forestal.pdf. Consultado 25/05/2015

Montoya Restrepo, L., Martínez Vianchá, L., \& Peralta Ballesteros, J. (2005). Análisis de variables estratégicas para la conformación de una cadena productiva de quinua en Colombia. INNOVAR, 103-119.Disponible en: http://revistas.unal.edu.co/index.php/ innovar/article/view/34. Consultado 2505-2015

Naciones Unidas. (2013). Resolución 66/288. El futuro que queremos. Disponible en: http://www.un.org/es/sustainablefuture/. Consultado 25/05/2015

Ortiz, N. R., Varela, O. E. T., Garcés, R. P., \& Ganzález, Y. R. (2015, January). PUB-
LIC POLICY AS A SUPPORT MECHANISM FOR RURAL DEVELOPMENT. In Global Conference on Business \& Finance Proceedings (Vol. 10, No. 1, p. 666). Institute for Business \& Finance Research. Disponible en:

http://search.proquest.com/openview/ 629d3aae0f365650afc367997f3f5264/1?pqorigsite $=$ gscholar. Consultado 25/05/2015

Sepúlveda S., S. (2008). Gestión del desarrollo sostenible en territorios rurales: métodos para la planificación. San José, Costa Rica. Disponible en: https://www.google.com.co/url?sa=t\&rct $=\mathrm{j} \& \mathrm{q}=\&$ esrc $=\mathrm{s} \&$ source $=$ web\&cd $=1 \& \mathrm{cad}$ $=$ rja\&uact $=8 \&$ ved $=0 \mathrm{CBsQFjAA} \& u r l=$ http $\% 3 \mathrm{~A} \% 2 \mathrm{~F} \% 2 \mathrm{Fwww}$.territorioscentro americanos.org\% $\%$ Fexperiencias $\% 2 \mathrm{FDoc}$ uments $\% 2$ FPlanificaci $\% 25 \mathrm{C} 3 \% 25 \mathrm{~B} 3 \mathrm{n} \% 25$ 20 y\% 2520Gesti\%25C3\%25B3n\%2520de\% 2520Territorios.pdf\&ei=16djVbjTDMnms ATlloDQBA\&usg=AFQjCNFfyeZcDrSYl YxphcW8FVUk0dRTwA\&sig2=u0qcaAH B2nrW_eklBpnr6A\&bvm=bv.93990622, d.cWc

Shabanova, L. B., Ismagilova, G. N., Salimov, L. N., \& Akhmadeev, M. G. (2015). PEST-Analysis and SWOT-Analysis as the Most Important Tools to Strengthen the Competitive Advantages of Commercial Enterprises.Mediterranean Journal of Social Sciences, 6(3), 705. Disponible en: http://www.mcser.org/journal/index.php/ mjss/article/view/6606. Consultado 25-052015

Universidad Nacional de Colombia - Sede Bogotá. (2012). GUIA ANALISIS PEST. Bogotá: Disponible en:

www.bogota.unal.edu.co/anterior/objects/ docs/.../Guia_Analisis_PEST.pdf. Consultado 25-05-2015 